\title{
AN EQUIVARIANT VERSION OF GROMOV'S THEOREM
}

\author{
BY EDWARD BIERSTONE ${ }^{1}$ \\ Communicated by Gian-Carlo Rota, January 22, 1973
}

In this note we announce an equivariant version of the theorem of Gromov [2], [3], [7] concerning the classification of smooth sections of a differentiable fibre bundle whose $r$-jets satisfy an "intrinsic differential inequality". The development of Gromov's theorem began with the Smale-Hirsch theory of immersions [8], [5], which was clarified and generalized by Phillips [6], Haefliger and Poenaru [4] and Gromov. Phillips' submersion theorem makes clear the essential role played by the assumption that the source manifold is nonclosed (i.e., no compact component meets the boundary); in fact the immersion theorem in positive codimension can be deduced from the submersion theorem using the (nonclosed) normal bundle of the source manifold in the target.

I would like to thank my thesis advisor, Richard S. Palais, for his encouragement and generous advice.

Preliminaries on $G$-fibre bundles. Throughout this paper $G$ denotes a compact Lie group. A $G$-manifold is a differentiable $\left(C^{\infty}\right)$ manifold $X$ together with a differentiable action of $G$ on $X$. Let $X$ be a $G$-manifold and $p: E \rightarrow X$ a (locally trivial) differentiable fibre bundle. If there is a differentiable action of $G$ on $E$ such that each $g \in G$ operates as a bundle map over the given map $g: X \rightarrow X$, then we say that $p: E \rightarrow X$ is a (differentiable) $G$-fibre bundle (when $p$ has a specified Lie structure group, bundle maps are understood to be induced by principal bundle maps). For example, the projection $p: X \times Y \rightarrow X$ from a product of $G$-manifolds with the diagonal action is equivariant, and $G$ acts as a group of bundle maps if we consider $p$ a trivial fibre bundle with structure group $G$.

A differentiable $G$-fibre bundle $p: E \rightarrow X$ is called $G$-locally trivial if for each $x \in X$ there is a $G_{x}$-invariant neighbourhood $U_{x}$ of $x\left(G_{x}\right.$ is the isotropy subgroup of $x$ ) such that $p \mid U_{x}$ is differentiably $G_{x}$-equivariantly equivalent to the trivial $G_{x}$-fibre bundle $U_{x} \times p^{-1}(x)$. $G$-local triviality allows us to work equivariantly in local coordinates. Though differentiable

AMS (MOS) subject classifications (1970). Primary 57E15, 58C25, 58D10; Secondary 55D10, 58A20.

Key words and phrases. $G$-manifold, orbit bundle, handle bundle, $G$-fibre bundle, nonclosed manifold, $r$-jet, immersion, submersion.

${ }^{1}$ The author was supported at Brandeis University by a National Research Council of Canada Postgraduate Scholarship. 
$G$-fibre bundles are not in general $G$-locally trivial, we do have the following theorem [1].

THEOREM 1. Let $p: E \rightarrow X$ be a differentiable $G$-fibre bundle with either Lie structure group or compact fibre. Then $p$ is $G$-locally trivial.

$G$-manifolds with nonclosed orbit bundles. Let $X$ be a $G$-manifold and $(H)$ an orbit type of $X$; i.e., the conjugacy class of the isotropy subgroup $H$ of a point in $X$. The union $X_{(H)}$ of all orbits of type $(H)$ is a $G$-invariant differentiable submanifold of $X$. We call $X_{(H)}$ an orbit bundle of $X$ since it is in a canonical way a right fibre bundle with the orbits of type $(H)$ as fibres.

We recall (see Wasserman [9, pp. 144-150]) that a $G$-manifold possesses an invariant decomposition into bundles of handles over orbits, just as a manifold possesses a handle decomposition. Let $G x$ be an orbit in the $G$-manifold $X$, and let $V, W$ be Riemannian $G$-vector bundles over $G x$. The bundle

$$
D(V) \oplus D(W)=\{(x, y) \in V \oplus W \mid\|x\| \leqq 1,\|y\| \leqq 1\}
$$

is called a handle bundle with index $=\operatorname{dim} V$. Suppose $Z, Y$ are invariant submanifolds of $X$, and $\varphi: S(V) \oplus D(W) \rightarrow \partial Z$ is an equivariant embedding, where

$$
S(V) \oplus D(W)=\{(x, y) \in V \oplus W \mid\|x\|=1,\|y\| \leqq 1\} .
$$

If $Y$ arises from the disjoint union of $Z$ and $D(V) \oplus D(W)$ by identifying each $p \in S(V) \oplus D(W)$ with $\varphi(p) \in \partial Z$, then we say $Y$ is obtained by "attaching the handle bundle $D(V) \oplus D(W)$ to $Z$ via $S(V) \oplus D(W)$ " (here and elsewhere we ignore problems of rounding corners).

Recall (Phillips [6, pp. 176-177]) that a manifold is nonclosed if and only if it possesses a handle decomposition with no handles of index equal to the dimension of the manifold. In the hypotheses of Gromov's theorem the base of the fibre bundle is nonclosed, and the transverse discs of the handles in a decomposition with no handles of top index give room to make deformations inside the handles needed to prove the theorem. In the case of $G$-manifolds, though a handle bundle over an orbit may not be of top index, the action of the isotropy subgroup of a point in the orbit on the transverse disc of the fibre over that point imposes symmetry conditions restricting our freedom to make equivariant deformations. But if all orbit bundles of a $G$-manifold are nonclosed, there is a handle bundle decomposition which recovers this freedom.

We say that a handle bundle $D(V) \oplus D(W)$ (over an orbit $G x$ ) with attaching bundle $S(V) \oplus D(W)$ is good if the fibre $W_{x}$ of $W \cong G \times_{G_{x}} W_{x}$ 
over a point $x$ on the orbit $G x$ has a one-dimensional subspace on which the isotropy subgroup $G_{x}$ acts trivially.

Let $X$ be a $G$-manifold and $\left\{\left(H_{j}\right) \mid j \in J\right\}$ the orbit types of $X$. We denote by $X^{H_{j}}$ the fixed point set of $H_{j}$, and by $X^{\left(H_{j}\right)}$ the saturation $G \cdot X^{H_{j}}$ of $X^{H_{j}}$. An invariant component of $X$ is the inverse image under the quotient map $X \rightarrow X / G$, of a component of the orbit space $X / G$. For each $j \in J$ let $\left\{X_{i}^{j} \mid i \in I(j)\right\}$ be the invariant components of $X^{\left(H_{j}\right)}$; the $X_{i}^{j}$ are closed subspaces of $X$. We partially order $\left\{X_{i}^{j} \mid i \in I(j), j \in J\right\}$ by inclusion. The minimal elements of this partially ordered set are precisely the (topologically) closed invariant components of the orbit bundles $X_{(\boldsymbol{H})}$ of $X$ (so are invariant submanifolds of $X$ ).

THEOREM 2. The following conditions are equivalent:

(a) All minimal elements of the partially ordered set $\left\{X_{i}^{j}\right\}$ are nonclosed.

(b) All orbit bundles $X_{(\mathrm{H})}$ of $X$ are nonclosed.

(c) There is a decomposition of $X$ into good handle bundles.

The main theorem. We consider a $G$-locally trivial differentiable $G$-fibre bundle $p: E \rightarrow X$ which is naturally associated to the differentiable structure of the $G$-manifold $X$ in the following sense. Let $\mathscr{D}_{G}(X)$ be the pseudogroup of $G$-diffeomorphisms between invariant open subsets of $X$. We assume there is an extension of $\mathscr{D}_{G}(X)$ to $\mathscr{D}_{G}(E)$; i.e. a map $\Phi: \mathscr{D}_{G}(X) \rightarrow \mathscr{D}_{G}(E)$ such that:

1. If $(f: U \rightarrow V) \in \mathscr{D}_{G}(X)$, then $\Phi(f)$ is an equivariant diffeomorphism $p^{-1}(U) \rightarrow p^{-1}(V)$ and $p \circ \Phi(f)=f \circ p \mid p^{-1}(U)$.

2. $\Phi(\operatorname{id}(U))=\operatorname{id}\left(p^{-1}(U)\right)$.

3. $\Phi(f \circ g)=\Phi(f) \circ \Phi(g)$ when $f \circ g$ is defined.

We assume, moreover, that the extension $\Phi$ is continuous in the sense that for every invariant open subset $U$ of $X, \Phi: \mathscr{D}_{\text {iff }}(U) \rightarrow \mathscr{D}_{\text {iff }}\left(p^{-1}(U)\right)$ is continuous (in the $C^{\infty}$ topology), where $\mathscr{D i f f}_{G}(U)$ is the group of equivariant diffeomorphisms from $U$ onto itself. Examples are the trivial bundle $E=X \times Y$, where $X$ and $Y$ are $G$-manifolds, with the "trivial" extension $\Phi$ given by $\Phi(f)(x, y)=(f(x), y)$, and the tangent bundle $T X$ of a $G$-manifold $X$, with the actions of $G$ and $\mathscr{D}_{G}(X)$ on the total space given by the differentials of the actions on $X$.

Let $p^{r}: E^{r} \rightarrow X$ be the bundle of $r$-jets of $p$. Then $p^{r}$ is naturally a differentiable $G$-fibre bundle. Let $E_{G}^{r} \subseteq E^{r}$ be the $G$-invariant subspace (not subbundle) of $E^{r}$ comprising $r$-jets of "equivariant local sections" of $p$; i.e. equivariant sections defined on invariant open subsets of $X$, and let $p_{G}^{r}$ be the restriction of $p^{r}$ to $E_{G}^{r}$.

The extension $\Phi: \mathscr{D}_{G}(X) \rightarrow \mathscr{D}_{G}(E)$ naturally induces an extension $\Phi^{r}: \mathscr{D}_{G}(X) \rightarrow \mathscr{D}_{G}\left(E^{r}\right)$ as follows. Let $(f: U \rightarrow V) \in \mathscr{D}_{G}(X)$ and $\varphi$ be a germ of section of $p$ at $x \in U$. Then $\psi=\Phi(f) \circ \varphi \circ f^{-1}$ is a germ of 
section at $f(x)$, and we define $\Phi^{r}(f)\left(j_{x}^{r} \varphi\right)=j_{f(x)}^{r} \psi$. Note that $E_{G}^{r}$ is invariant under the action of $\mathscr{D}_{G}(X)$ via the induced extension $\Phi^{r}$.

Now the $r$-jet map induces a continuous map $j^{r}: \Gamma_{G}^{\infty}(p) \rightarrow \Gamma_{G}^{0}\left(p_{G}^{r}\right)$ from the space of $C^{\infty}$ equivariant sections of $p$ (with the $C^{\infty}$ topology) to the subspace $\Gamma_{G}^{0}\left(p_{G}^{r}\right) \subset \Gamma_{G}^{0}\left(p^{r}\right)$ of continuous equivariant sections of $p^{r}$ (with the $C^{0}$ topology) whose images lie in $E_{G}^{r}$.

Finally let $E_{\omega}^{r}$ be an open $G$-subbundle of $E^{r}$ which is invariant under the action of $\mathscr{D}_{G}(X)$, and let $\Gamma_{G, \omega}^{\infty}(p) \subset \Gamma_{G}^{\infty}(p)$ be the subspace of equivariant sections $f: X \rightarrow E$ such that $j^{r} f(x) \in E_{\omega}^{r}$ for all $x \in X$. Let $E_{G, \omega}^{r}=E_{G}^{r} \cap E_{\omega}^{r}$ and $p_{G, \omega}^{r}=p^{r} \mid E_{G, \omega}^{r}$. We see that $j^{r}$ induces a continuous $\operatorname{map}^{r}: \Gamma_{G, \omega}^{\infty}(p) \rightarrow \Gamma_{G}^{0}\left(p_{G, \omega}^{r}\right)$.

THEOREM 3. If $X$ satisfies the conditions of Theorem 2, then

$$
j^{r}: \Gamma_{\mathbf{G}, \omega}^{\infty}(p) \rightarrow \Gamma_{G}^{0}\left(p_{G, \omega}^{r}\right)
$$

is a weak homotopy equivalence.

REMARKS ON PROOF. The theorem is first proved for a disc bundle over an orbit. Then suppose that $X_{1} \subset X_{2} \subset X$ are compact submanifolds of $X$ of the same dimension as $X$ such that $X_{2}$ is obtained from $X_{1}$ by attaching a good handle bundle. We have a commutative diagram

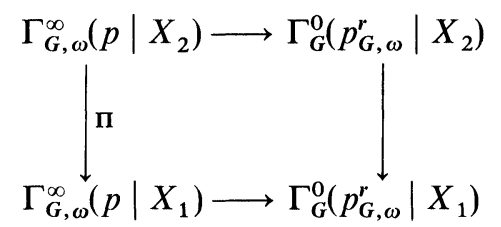

where the vertical maps are given by restriction. We prove that the vertical arrows are Serre fibrations, and then, by induction on the index of the handle bundle (again using its goodness) that if the lower arrow is a weak homotopy equivalence, then so is the upper. A good handle bundle decomposition of $X$ gives towers of weak homotopy equivalent fibrations, and the theorem follows by taking inverse limits of these towers. The use of good handle bundles allows us to prove that $\Pi$ is a fibration in the same way as in Gromov's theorem, when $G=1$. The differences in the remainder of the proof from the usual proofs of Gromov's theorem are mainly technical, arising from the facts that $p_{G}^{r}$ is not in general a bundle and that translation of a linear $G$-space is not in general equivariant.

We remark as well that a relative version of the theorem can be proved at no extra cost, and that the theorem specializes to Gromov's theorem (as given by Poenaru [7]) when $G=1$.

Equivariant submersion and immersion theorems. Let $X$ and $Y$ be $G$ manifolds. We consider the trivial $G$-fibre bundle $p: E=X \times Y \rightarrow X$ 
with the trivial extension $\Phi: \mathscr{D}_{G}(X) \rightarrow \mathscr{D}_{G}(E)$. Let $E_{\omega}^{1}$ be the open $G$ - and $\mathscr{D}_{G}(X)$-invariant subbundle of $E^{1}$ consisting of 1 -jets of maximal rank.

If $\operatorname{dim} X \geqq \operatorname{dim} Y$, then $\Gamma_{G, \omega}^{\infty}(p)=\operatorname{Sub}_{G}(X, Y)$, the space of equivariant submersions of $X$ into $Y$ with the $C^{\infty}$ topology, and $\Gamma_{G}^{0}\left(p_{G, \omega}^{1}\right)=$ $\operatorname{Max}_{G}^{*}(T X, T Y)$, the space of equivariant bundle maps $F: T X \rightarrow T Y$ which have maximal rank on each fibre, and such that for each $x \in X$, $F_{x} \mid T(G x)_{x}$ is given by the differential of the map $g x \rightarrow g f(x)$ of $G x$ onto $G f(x)$ (where $f: X \rightarrow Y$ is the map induced by $F$ ). The main theorem gives an equivariant version of Phillips' theorem [6]:

THEOREM 4. If $X$ satisfies the conditions of Theorem 2, then the differential d: $\operatorname{Sub}_{G}(X, Y) \rightarrow \operatorname{Max}_{G}^{*}(T X, T Y)$ is a weak homotopy equivalence.

We now assume that $\operatorname{dim} X<\operatorname{dim} Y$ and that $X$ may be a closed $G$-manifold. Let $\operatorname{Imm}_{G}(X, Y)$ be the space of equivariant immersions $X \rightarrow Y$, and $R_{G}^{*}(T X, T Y)$ the space of bundle maps $F: T X \rightarrow T Y$ which are injective on each fibre and, as above, $F_{x} \mid T(G x)_{x}$ is given by the differential of the induced map from $G x$ onto $G f(x)$. Using the notation preceding Theorem 2:

THEOREM 5. If the dimension of the fixed point set of $H_{j}$ in each minimal element $X_{i}^{j}$ is strictly less than the dimension of each component of $Y^{H_{j}}$, then the differential induces a weak homotopy equivalence $d: \operatorname{Imm}_{G}(X, Y) \rightarrow$ $R_{G}^{*}(T X, T Y)$.

The hypotheses on $X$ ensure that the total space $E$ of the normal bundle $v$ of an equivariant immersion of $X$ in $Y$ has a good handle bundle decomposition. Except for a technical difficulty in showing that restriction to the zero section gives a fibration $\operatorname{Max}_{G}^{*}(T E, T Y) \rightarrow R_{G, v}^{*}(T X, T Y)$, where $R_{G, v}^{*}(T X, T Y) \subset R_{G}^{*}(T X, T Y)$ is the subspace of bundle monomorphisms with complementary bundle $v$, the immersion theorem follows from the submersion theorem as in Phillips [6, Theorem 8.4, p. 196].

\section{REFERENCES}

1. E. Bierstone, The equivariant covering homotopy property for differentiable G-fibre bundles, J. Differential Geometry (to appear).

2. M. L. Gromov, Stable mappings of foliations into manifolds, Izv. Akad. Nauk SSSR Ser. Mat. 33 (1969), 707-734 = Math. USSR Izv. 3 (1969), 671-694. MR 41 \# 7708.

3. A. Haefliger, Lectures on the theorem of Gromov, Proceedings of Liverpool Singularities Symposium II, Lecture Notes in Math., vol. 209, Springer-Verlag, Berlin, 1971.

4. A. Haefliger and V. Poenaru, La classification des immersions combinatoires, Inst. Hautes Études Sci. Publ. Math. No. 23 (1964), 75-91. MR 30 \#2515.

5. M. Hirsch, Immersions of manifolds, Trans. Amer. Math. Soc. 93 (1959), 242-276. MR 22 \# 9980.

6. A. Phillips, Submersions of open manifolds, Topology 6 (1967), 171-206. MR 34 \# 8420 . 
7. V. Poenaru, Homotopy theory and differentiable singularities, Manifolds-Amsterdam 1970, Lecture Notes in Math., vol. 197, Springer-Verlag, Berlin, 1971. MR 44 \# 2250.

8. S. Smale, The classification of immersions of spheres in Euclidean spaces, Ann. of Math. (2) 69 (1959), 327-344. MR 21 \#3862.

9. A. G. Wasserman, Equivariant differential topology, Topology 8 (1969), 127-150. MR 40 \#3563.

Department of Mathematics, State University of New York, Stony Brook, New YORK 11790

Current address: Department of Mathematics, University of Toronto, Toronto, Ontario, Canada M5S 1A1 